\title{
Outer Halo Star Clusters: Comparisons for Cloud Clusters
}

\author{
J.E. Hesser, P.B. Stetson, S. van den Bergh
}

National Research Council of Canada, Herzberg Inst. of Astrophysics, Dominion Astrophysical Obs., Victoria, BC V8X 4M6, Canada

\section{Bolte, J.A. Johnson}

U. of California, UCO/Lick Observatory, Santa Cruz, CA 95064, USA

\author{
W.E. Harris
}

McMaster University, Department of Physics and Astronomy, Hamilton, ON L8S 4M1, Canada

\section{D.A. VandenBerg}

University of Victoria, Department of Physics and Astronomy, Victoria, $B C V 8 W 3 P 6$, Canada

\section{R.A. Bell}

University of Maryland, Department of Astronomy, College Park, MD 0742-2421, USA

\author{
H.E. Bond, L.E. Fullton
}

STScI, 3700 San Martin Dr., Baltimore, MD 21218, USA

G.G. Fahlman, H.B. Richer

University of British Columbia, Department of Physics and Astronomy, Vancouver, BC V6T 1Z4, Canada

\begin{abstract}
Color-magnitude diagrams reaching from the giant branches to $\mathrm{V}_{\lim } \sim 27.0$, or about three magnitudes fainter than the turnoff, have been obtained in V and I with WFPC2 on HST for NGC 2419, Pal 3, $\mathrm{Pal} 4$ and Eridanus, whose relative ages are discussed.
\end{abstract}

\section{Formation of the Milky Way Halo}

The ages, chemistry and other properties of outer Galactic halo star clusters make them powerful objects for comparison with the oldest star clusters of the Magellanic Clouds (e.g., see the individual papers by Geisler, Mighell, Da Costa, Olsen, and Johnson in these proceedings) and other Local Group galaxies. They also constrain the chronology and duration of early cluster formation by contributing to understanding the relative roles of rapid collapse and multi-Gigayear 
accumulation of many fragments. We seek to understand if the more remote globular clusters with unusually red horizontal branches $(\mathrm{HBs})$ for their $[\mathrm{Fe} / \mathrm{H}]$ - the "second-parameter" clusters - are systematically younger than innerhalo objects, where the second-parameter phenomenon is weaker. Direct age measurements can be made only with color-magnitude diagram (CMD) photometry reaching well below the main sequence turnoff, thus avoiding dependence upon HB morphology, which is sensitive to many parameters besides age.

WFPC2 F555W ('V') and F814W ('I') photometry processed through the suite of DAOPHOT/ALLSTAR/ALLFRAME codes cleanly defines CMDs for the following clusters from their giant and HBs down through their upper main sequences: Pal 3: $\left(\mathrm{M}_{\mathrm{V}}=-5.2, \mathrm{R}_{\odot} \sim 87 \mathrm{kpc}\right),[\mathrm{Fe} / \mathrm{H}] \sim-1.6 ; \mathrm{Pal} 4:\left(\mathrm{M}_{\mathrm{V}}=\right.$ $\left.-5.8, \mathrm{R}_{\odot} \sim 98 \mathrm{kpc}\right),[\mathrm{Fe} / \mathrm{H}] \sim-1.3 ;$ Eridanus: $\left(\mathrm{MV}_{\mathrm{V}}=-4.8, \mathrm{R}_{\odot} \sim 78 \mathrm{kpc}\right),[\mathrm{Fe} / \mathrm{H}]$ $\sim-1.4$; and NGC 2419: $\left(\mathrm{M}_{\mathrm{V}}=-9.5, \mathrm{R}_{\odot} \sim 88 \mathrm{kpc}\right),[\mathrm{Fe} / \mathrm{H}] \sim-2.1$. Each cluster exhibits a population of blue stragglers. Pal 3 contains several RR Lyrae variables.

\section{Relative Ages}

Accurate knowledge of abundances, and abundance ratios, is essential for both relative and absolute age determinations. Unfortunately, even in nearby Galactic globular clusters (whose giants are sufficiently bright for high-dispersion analyses), there is considerable dispersion among studies. For the outer-halo objects, abundance information is qualitatively different, as it is based upon lowdispersion spectra (often only of the calcium triplet in a few giants) and various indirect photometric indicators.

NGC 2419 and the nearby cluster M92 have indistinguishable ages, providing there are no unusual ratios of abundances (e.g., $[\alpha / \mathrm{Fe}]$ ) in either of these clusters (Harris et al. 1997). Thus, the earliest star (or globular cluster) formation began at essentially the same time everywhere in the Galactic halo throughout a region now almost $200 \mathrm{kpc}$ in diameter.

Two relative age indicators, $\Delta V_{\mathrm{TO}}^{\mathrm{HB}}$ and $\Delta(V-I)$, suggest that all three second-parameter clusters are younger than their nearby counterparts by 1.5 to 2 Gyr (Stetson et al. 1999), which is in the same sense and approximately the same size predicted by HB star modeling. However, if we ask what other parameters would have to be different for the "second parameter" clusters to have the same ages as the inner halo clusters, we find that an overestimation of the former's abundances by only $0.3 \mathrm{dex}$ (either in $[\mathrm{Fe} / \mathrm{H}]$ or $[\alpha / \mathrm{Fe}]$ would be required. If Pal 3, Pal 4 and Eri were to have primordial abundances of $[\alpha / \mathrm{Fe}]$ $\sim 0.0$, as Brown et al. (1997) found for Rup 106 and Pal 12, that would erase half of the inferred age difference between them and the inner halo clusters.

\section{References}

Brown, J.A., Wallerstein, G., \& Zucker, D. 1997, AJ, 114, 180

Harris, W.E., et al. 1997, AJ, 114, 1030

Stetson, P.B., et al. 1999, AJ, 117, 247 